Original article

\title{
Knowledge and awareness regarding HPV infection and PAP smear screening in reproductive aged females of rural India
}

\author{
Manisha Sharma ${ }^{1, *}$, Chandra Shekhar Kapoor ${ }^{2}$ \\ Department of Life Science, Pacific Academy Of Higher Education And Research University, Udaipur, India
}

\section{A R T I C L E I N F O}

\section{Keywords:}

Cervical cancer

Human papilloma virus (HPV)

Screening

Awareness

PAP smear

\begin{abstract}
A B S T R A C T
Purpose: The purpose of the study was to document the knowledge and awareness of HPV infection and Pap smear among the study women and to conduct screening with Pap smear cytology and HPV testing. The study also aimed to further perform a correlation analyses of HPV prevalence with demographic data.

Methods: The present study collected demographic, epidemiologic and clinical information of women of aged group 20-60 years with gynaecological abnormalities who attended the gynaecology and cancer OPD at a tertiary care health center.

Results: Majority of the study participants were young married ladies and did not have any formal education $(85.5 \%)$. Mean (SD) age at the time of first pregnancy among the study subjects was 16.8 (2.7) years. Majority of the study participants were below poverty line (88.2\%). A total of $68.6 \%$ of the study subjects had an HPV infection. Among those that had an HPV infection, majority had HPV 16 infection (95.4\%). In the remaining 4.6\%, HPV 18 was present. Findings show that with increasing age, HPV prevalence increases. Also, early age at marriage, lack of education, increased parity, early age at first pregnancy, poor sanitation, use of tobacco and belonging to below poverty line were significant risk factors for HPV infection in this cohort.

Conclusion: The study reported a high prevalence of HPV infection in rural women attending the Gynaecology and Cancer OPD of a tertiary care hospital. Several important risk factors for the HPV infection were also identified. These were early age at marriage, lack of education, increased parity, early age at first pregnancy, poor sanitation, use of tobacco and belonging to below poverty line. These findings call for more efforts to increase awareness pertaining to HPV infection and cervical cancer among rural women.
\end{abstract}

\section{Introduction}

In developing countries, cervical cancer is the second most commonly diagnosed cancer with 77000 (95\% CI; 68000-96000) incident cervical cancer cases in India in 2016 and 288000 (247000-342000) prevalent cases. ${ }^{1-4}$ Its third leading cause of cancer-related death after breast and lung cancers. ${ }^{1,2}$ The age-standardized incidence rate for cervical cancer varied 2.8 times in females across the states of India in 2016. ${ }^{1,3,4}$ Cervical cancer was the second leading cause of cancer deaths for females in 12 Indian states. ${ }^{1,3-5}$

The estimated risk of cervical cancer is calculated out to be approximately 1 in 53 Indian women during their lifetime compared with 1 in 100 women in developed nations. ${ }^{6-8}$ In India, cervical cancer is the most common cancer accounting for $25.9 \%$ of new cancer cases and $23.3 \%$ of all cancer related deaths. ${ }^{9-11}$ Statistically, yearly approximately 90,000 new cases of cervical cancer are noted. The incidence in India is 45 per one lakh women. ${ }^{3,8,9,11}$ Preinvasive lesions of the cervix if diagnosed early and treated have a higher cure rate but advanced disease is frequently incurable with very unpleasant consequences. ${ }^{13,14}$ It is the only type of cancer which can be prevented. However, prevention of cervical cancer remains merely an illusion due to poor health system infrastructure and low on resources in developing countries like India. Nevertheless, majority of women never undergone pelvic examination as reported in various screening programs with low coverage. In developed countries that employ intensive cervical cancer screening programs (that is, Pap smear), the incidence and mortality rates of cervical cancer have been reduced by roughly $70-80 \% .{ }^{10,11}$

Due to lack of data on cervical cancer prevalence in regions near to Delhi NCR, this study would give a prevalence rate and incidence rate of HPV infection leading to cervix cancer. The gaps in cervical cancer

\footnotetext{
${ }^{*}$ Corresponding author.

E-mail addresses: manishabhardwaj17@gmail.com (M. Sharma), drcskapoor@yahoo.co.in (C.S. Kapoor).

${ }^{1}$ First Author (Main author).

${ }^{2}$ Co author.
} 
research have been widening in recent decades as screening and effective preventive strategies have led to a dramatic decline in the prevalence and mortality rates in developed countries. There are some reports that are available for HPV prevalence in Delhi region but no data available for its adjacent cities like Gurgaon (Haryana). Haryana has different and varying socio-economic, educational and cultural hesitations. In order to introduce importance of vaccination, promote its necessity and to take up screening and management of the disease, it becomes really important to understand and draw pattern on prevalence of HPV in the specific population, which is predominant objective of the present study. India has a high burden of cervical cancer, but there is wide disparity in the incidence within the individual states and geographical locations. ${ }^{1-5,15}$ The cancer registries are existing, but they cover a limited population and therefore we do not have robust updated data from specific regions of India on the epidemiology of cervical cancer; its risk factors; prevalence of HPV infections and on appropriate preventive and treatment strategies. This present study has been taken up to understand causes, prevalence of cervical cancer from rural areas of Delhi NCR since there is a significant variation in social life, sexual life and geographic aspects.

\section{Material and methods}

Study population: Women aged $20-60$ years who had indications of cervical malignancy and those that attended the gynaecology and cancer OPD in a tertiary care center/hospital of Haryana, India.

Tools: A pretested questionnaire was prepared that had critical parameters listed down for effective study and analysis. These questionnaires were asked to fill by the study participants and included questions on: Age and Marital status; Age of marriage and Parity; ever screened for cervical cancer; When was the last screening test done; Year completed in formal education; Hormonal contraceptives use; What are the abnormalities you are facing and since when; Tobacco/ smoking use; Sanitation; Economic status.

Techniques: Pap smear; visual inspection with acetic acid; clinical biopsies (in consultation with gynaecologist); DNA isolation from biopsy samples (phenol/chloroform method); HPV serotyping (PCR primer specific).

Study design: A prospective observational descriptive study. A prior permission from the Institutional Ethical committee was obtained. This study did not impose financial burden on study participants. A well informed consent in writing was received from the study subjects before conducting the study. All participants were ensured confidentiality. The study mainly included a descriptive survey. In this design, a questionnaire was prepared, pretested and asked to the patients coming to gynaecologist and consulting cancer department. All the information collected was kept confidential. After screening, the diagnosis reports were collected from doctors, e.g. Pap smear test or visual inspection with acetic acid. Simultaneously HPV serotyping was done by PCR under laboratory controls. After the disease diagnosis, information was collected on treatment strategies.

\subsection{Statistical analysis}

Data entry, data cleaning \& data analyses were done by Statistical Package for Social Sciences software (version 20.0; SPSS). Output indicators were analysed through the software and results were subsequently presented in form of tables \& graph. Mean (SD; standard deviation) were calculated for continuous variables and proportions for categorical variables. Proportions were compared using chi-square whereas means were compared using independent $t$-test. For all comparisons, the p-value of $<0.05$ was considered statistically significant. Sample size of 196 was calculated based on assuming the prevalence of HPV infection to be $60 \%$, absolute precision of $7 \%$, and $90 \%$ power. We further assumed a non-response rate of $10 \%$. Hence, the total sample size was 220 . We used the formula $4 \mathrm{PQ} / \mathrm{d}^{2}$ where $\mathrm{P}=$ prevalence;
Table 1

Distribution of HPV prevalence by age, age at marriage \& at first pregnancy of the participants.

\begin{tabular}{|c|c|c|c|}
\hline Age (in years) & Total subjects & N (\%) & P-value \\
\hline $20-30$ & 62 & $30(48.4)$ & \multirow[t]{5}{*}{$0.0001^{\mathrm{a}}$} \\
\hline $30-40$ & 72 & $41(56.9)$ & \\
\hline $40-50$ & 70 & $64(91.4)$ & \\
\hline$>50$ & 16 & $16(100.0)$ & \\
\hline Age at marriage (in years) & Total subjects & N (\%) & \\
\hline $11-18$ & 194 & $138(71.1)$ & \multirow[t]{3}{*}{$0.01^{\mathrm{a}}$} \\
\hline $18-22$ & 26 & $13(50.0)$ & \\
\hline Age at 1st pregnancy (in years) & Total subjects & $\mathrm{N}(\%)$ & \\
\hline $13-18$ & 178 & $126(70.7)$ & \multirow[t]{2}{*}{$0.04^{\mathrm{a}}$} \\
\hline 19-23 & 42 & 25 (59.5) & \\
\hline
\end{tabular}

a Statistically significant.

$\mathrm{Q}=100-\mathrm{P} ; \mathrm{d}=$ absolute precision

\section{Results}

The mean (SD) age of the participants was 37.8 (9.2) years and most of the study participants were aged between 11 and 18 years at the time of their marriage (88.2\%) (Table 1 ). Mean (SD) age at the time of first pregnancy among the study subjects was 16.8 (2.7) years. Only around $19 \%$ were aged between 19 and 23 years at the time of their first pregnancy (Table 1). The mean (SD) age of the participants at the time of their first PAP smear testing was 37.6 (9.0) years. A total of $29.1 \%$ participants had their first PAP smear between the age range of 20-30 years; $32.7 \%$ in the age group of $30-40$ years; $31.8 \%$ in the age range of 40-50 years (Table 1 ). All the study subjects were married $(100.0 \%)$ and had a parity of 1-3 (51.8\%) followed by those with the parity of 4 or more $(46.4 \%)$. All of the study participants were not vaccinated with HPV vaccine before screening. Among the study subjects, women who were having tobacco or were smoking, had HPV prevalence of around 98.4\%; and for those who were not having any of these had HPV prevalence reduced to $31.6 \%$ (Table 2 ) ( $\mathrm{P}=0.0001)$. Among the study subjects, women who had no education had HPV prevalence of 70.7\%; for women who were in class $1-10^{\text {th }} \mathrm{HPV}$ prevalence was $60 \%$; and for women who had higher education i.e. greater than that of class 10th, none had HPV (Table 2) $(\mathrm{P}=0.036)$. The data is showing clearly that the patients with no education have higher no. of affected patients.

Table 2

Distribution of HPV prevalence by education status, parity, sanitation level and tobacco/smoking habit.

\begin{tabular}{llll}
\hline Education status & Total subjects & $\mathrm{N}(\%)$ & P-value \\
\hline $\begin{array}{l}\text { No education } \\
\text { Class } 1-10^{\text {th }}\end{array}$ & 188 & $133(70.7)$ & 0.036 \\
Class $>10$ th & 30 & $18(60.0)$ & \\
No. of parity & 2 & $0(0.0)$ & \\
0 & 4 & $1(25.0)$ & $0.0001^{\mathrm{a}}$ \\
$1-3$ & 114 & $60(52.6)$ & \\
$\geq 4$ & 102 & $90(88.2)$ & \\
Sanitation level & & $1(25.0)$ & $0.02^{\mathrm{a}}$ \\
Good & 4 & $150(69.4)$ & \\
$\begin{array}{l}\text { Poor } \\
\text { Tobacco/smoking habit }\end{array}$ & 216 & $120(98.4)$ & $0.0001^{\mathrm{a}}$ \\
Yes & 122 & $31(31.6)$ & \\
$\begin{array}{l}\text { No } \\
\text { Socioeconomic status }\end{array}$ & 98 & $140(72.2)$ & $0.002^{\mathrm{a}}$ \\
Below poverty line & 194 & $11(42.3)$ & \\
Average & 26 & $0(0.0)$ & \\
Good & 0 & & \\
\hline
\end{tabular}

a Statistically significant. 
Table 3

Distribution of study participants according to use of contraceptives, or have knowledge/understanding of PAP smear, types of HPV infection and presenting features reported.

\begin{tabular}{ll}
\hline Number of abortions & $\mathrm{N}(\%)$ \\
\hline 0 & $108(49.1)$ \\
$1-2$ & $108(49.1)$ \\
$\geq 3$ & $4(1.8)$ \\
Use of contraceptives & \\
Yes & $2(0.9)$ \\
No & $218(99.1)$ \\
Hemorrhage & $160(72.7)$ \\
No & $60(27.3)$ \\
Heard about PAP testing & $14(6.4)$ \\
Yes & $206(93.6)$ \\
No & \\
Understand the importance of PAP testing & $5(2.3)$ \\
No & $215(97.7)$ \\
HPV infection & $151(68.6)$ \\
Yes & $69(31.4)$ \\
No & \\
HPV serotype 16 & $144(95.4)$ \\
HPV 18 & $7(4.6)$ \\
Genital warts concurrence & $160(72.7)$ \\
Absent & $60(27.3)$ \\
Pbnormalities reported & \\
Menopause & $179(81.4)$ \\
\hline & $41(18.6)$ \\
\hline
\end{tabular}

Among the study subjects, women who below poverty line had HPV prevalence of $72.2 \%$; for subjects whose socioeconomic status was average, HPV prevalence was reduced to $42.3 \%$ and for those whose socioeconomic status was good, they did not had HPV. This shows that with increasing socioeconomic status HPV prevalence reduces $(\mathrm{P}=0.002)$ [Table 2]. Among the study subjects, women with good sanitation had HPV prevalence of $25 \%$ and those with poor sanitation had HPV prevalence of $69.4 \%(\mathrm{P}=0.02)$ [Table 2]. Majority of the study participants had not heard about PAP testing (93.6\%) and did not understand the importance of PAP testing (92.7\%). Among those that have heard about about PAP testing, a total of $28.6 \%$ of the study subjects had source of information as gynaecologist. Another 14.3\% had close relative(s) as the source of information.

Only a small proportion (0.9\%) reported use of contraception. Similar proportions of the study subjects had either no abortions (49.1\%) or between 1 and 2 abortions (49.1\%) (Table 3). Majority of the study participants complained of pain, white discharge, pain while intercourse and itching (81.4\%) (Table 3). A total of $68.6 \%$ of the study subjects had an HPV infection. Among those that had an HPV infection, majority had HPV 16 infection (95.4\%) while in the remaining 4.6\%, HPV 18 was present (Table 3). Majority of the study participants had presence of genital warts concurrently (72.7\%). Findings show that with increasing age HPV prevalence increases (Table 1).

\section{Discussion}

The results of our study reported low level of knowledge and awareness of cervical cancer, its screening and HPV among rural women cohort in Gurgaon, Haryana. In our study, 5-6\% of the participants had heard or understanding about Pap smear or cervical cancer. This incidence was quite lesser than to the several studies conducted in various South Asian countries have reported that between 50 and $85 \%$ of women were aware of cervical cancer. ${ }^{16-18}$ World Health Organization has concluded that screening for cervical cancer should be performed at least once for every woman in the target age group (30-49 years) when it is most beneficial. The recommended screening tests includes: HPV testing, cytology and visual inspection with acetic acid (VIA). The valuable holistic target approaches should be either "screenand-treat" or "screen, diagnose and treat". 19,20

The findings of this study reflect a poor level of awareness and knowledge towards sanitation level or personal hygiene among women. Educational level, occupation and higher income of the participants were determinants of adequate knowledge of cervical cancer, its screening and HPV in the Indian perspectives. ${ }^{21}$ We observed various factors like early age at marriage, lack of education, increased parity, early age at first pregnancy, poor sanitation, use of tobacco and belonging to below poverty line were significantly associated with HPV infection. These results are comparable with the study conducted in Madhya Pradesh, India. ${ }^{21}$

HPV 16 and 18 are the major etiologic infections for the cervical cancer. HPV 16, is predominantly found High-risk human population. In a cross-sectional study among 524 Indian women with symptoms of persistent vaginal discharge, intermenstrual bleeding, and postcoital bleeding, a wide spectrum of HPV infection has been seen in north Indian women, with the majority being HPV-16 type seen in $74.3 \%$ cases. ${ }^{22}$ In our study, a total of $68.6 \%$ of the study subjects those that had an HPV infection, 95\% had HPV-16 infection. This have been evident that using approach of organized cytological based pap smears, developed countries have substantially reduced the cervical cancer incidence by more than $70 \%$. This is made possible because of various preventive strategies including use of prophylactic vaccines and many secondary preventive strategies. Most of these interventions are currently not feasible in low income countries because of the already limited health care infrastructure and lack of awareness among women.

In our study, the mean age of the study participants was 38 years. The results of a meta-analysis showed that the highest prevalence of HPV occurs at the age of 25 years which could be related to changes in sexual behaviour. ${ }^{23}$ In another meta-analysis study, the bimodal distribution of cervical cancer in some regions has been studied. ${ }^{24}$ the bimodal distribution of cervical cancer in some regions has been studied i.e. age at first sexual intercourse, a sudden spurt (first peak) in HPV can be observed, followed by a plateau stage at adult age; then, after 45 years of age, the second peak again is noticed. ${ }^{24}$ In a developing country like India, carcinoma of cervix is seen at the prime age of 47 years. ${ }^{1-5}$ However, the disease can occur in both extremes of life.

Permanent infection with one of the high-risk types of HPV over time leads to the development of cervical intraepithelial neoplasia (CIN). ${ }^{25}$ The major mechanisms through which HPV contributes to carcinogenesis involve the activity of two viral oncoproteins, E6 and E7, which interfere with major tumor suppressor genes, P53 and $\mathrm{Rb}^{26,27}$ In addition, E6 and E7 are associated with changes in host DNA and virus DNA methylation that are associated with changes in key cellular pathways that regulate genetic integrity, cell adhesion, immune response, apoptosis, and cellular control. ${ }^{23,25,27,28}$

An increased risk of cervical cancer is observed in people with multiple sexual partners. ${ }^{23,28}$ This increase in risk is generally attributable to an increased risk of HPV infection. Also, early age at first intercourse is a risk factor for cervical cancer. ${ }^{29,30}$ We observed this age to be as low as 16.8 years in our study cohort. Several studies have shown that full-term pregnancy increases the risk of invasive cervical cancer. ${ }^{29,30}$ Moreover, high parity increases this risk of cancer in women. In a collaborative international epidemiological study of cervical cancer, a direct correlation was found between the risk of cervical cancer and parity ${ }^{30}$; there was an inverse association between the risk of cervical cancer and the age of the mother at first pregnancy.The risk of cervical cancer significantly increases with an increase in the number 
of pregnancies. In a cohort study with over 13 years of follow-up, parity was the predictor of CIN 3 especially among women with high risk HPV infection [32]. In our study, HPV prevalence was reported to be highest (90\%) among women with parity $\geq 4$.

In a collaborative international epidemiological study of cervical cancer, a direct correlation was found between the risk of cervical cancer and parity. It has been reported that the use of OC method for 5 years or more can double the risk of cancer. This was in contrast with findings of our study where this was found in less than $1 \%$ cases because of unawareness among rural women population.

To date, various studies have shown that smoking is one of the most important risk factors for CIN 3 and invasive cervical cancer. ${ }^{28,30}$ There is a link between the history of smoking or current smoking, with cervical cancer. We observed direct association of tobacco and smoking with HPV prevalence in $98 \%$ cases. In a large cohort study, the risk of cancer was reduced by $50 \%$ among those who stopped smoking for 10 years when compared with those who continued to smoke. ${ }^{27,28}$ Smoking can cause epigenetic changes in the epithelium of the cervix and contribute to the pathogenesis of neoplasia in the cervix and in many other parts of the body. ${ }^{26,27}$

This study has some limitations, as this study was conducted among women from rural background and hence the generalizability of the findings across India may not be scientific. Study was concentrated more on quantitative aspects by using the close ended questions.

\section{Conclusion and recommendations}

This study reported a high prevalence of HPV infection in women attending the Gynaecology and Cancer OPD of a tertiary care hospital. Based on evident facts, cervical cancer is easily preventable unlike most other cancers, with effective implemented programs to diagnose and manage at early stages. Ineffective utilization of prevention services by women in the 30-60 years (i.e. high-risk age groups) could be because of various factors such as unawareness of health professionals and women about the screening test, poor availability and accessibility of screening tools, compromised quality of care provided, and cultural/ behavioral restrictions. These findings call for more efforts to increase awareness pertaining to HPV infection and cervical cancer among women. Health education, barrier-specific counselling and outreach and community-based interventions should be ideal to provide an opportunity and to enhance the awareness of having cervical screening done. A well-designed health education program concerning cervical cancer and the benefits of screening and vaccination would increase the awareness among rural women. By imparting proper education, women can be empowered with methods of cervical cancer screening, and the availability of adequate timely management.

\section{Declaration of competing interest}

On behalf of all authors, the corresponding author states that there is no conflict of interest.

\section{Acknowledgement}

I would like to give my sincere gratitude to my supervisor Dr. Chandrashekhar Kapoor for providing their guidance and suggestions during my project. Also all the doctors and nursing staff for helping me during patients data collection.

\section{References}

1. Denny L. Cervical cancer: prevention and treatment. Discov Med. 2012;14:125-131.

2. Satija A. Cervical cancer in India. South Asia centre for chronic disease. . [Accessed February16, 2019]. Available from:. http://sancd.org/uploads/pdf/cervical_cancer. pdf.

3. Arbyn M, Castellsague X, DeSanjose S, et al. Worldwide burden of cervical cancer Ann Oncol. 2011;22:2675-2686

4. Yeole BB, Kumar AV, Kurkureet A, Sunny L. Population-based survival from cancers of breast, cervix and ovary in women in Mumbai. Asian Pac J Cancer Prev APJCP. 2004;5:308-315

5. ICO Information Centre on HPV and Cancer (Summary Report 2014-08-22).Human Papillomavirus and Related Diseases in India. 2014; 2014 [Google Scholar].

6. National Cancer Control Programme. . [Accessed July 16, 2014]. Available from: http://www.mohfw.nic.in/showfile.php?lid = 324 .

7. National Programme for Prevention and Control of Cancer. Diabetes, cardiovascular disease and stroke. . [Accessed July15, 2019]. Available from :. http://www.nrhmhp. gov.in/sites/default/files/files/NCD_Guidelines.pdf.

8. Gakidou E, Stella N, Ziad O. Coverage of cervical cancer screening in 57 countries: low average levels and large inequalities. PLoS Med. 2009;5:e132.

9. Aswathy S, Quereshi MA, Kurian B, Leelamoni K. Cervical cancer screening: current knowledge and practice among women in a rural population of Kerala, India. Indian J Med Res. 2012;136(2):205-210.

10. Sankaranarayanan R, Nene BM, Shastri SS, et al. HPV screening for cervical cancer in rural India. N Engl J Med. 2009;360:1385-1394.

11. Sankaranarayanan R, Esmy PO, Ramkumar R, et al. Effect of visual screening on cervical cancer incidence and mortality in Tamil Nadu: a cluster-randomised trial. Lancet. 2007;370:398-406.

13. Nandakumar A, Ramnath T, Chaturvedi M. The magnitude of cancer cervix in India Indian J Med Res. 2009;130(3):219-221.

14. National Centre for Disease Informatics Research, National Cancer Registry Programme. ICMR Three Year Report of Population Based Registries, 2009-2011 Bangalore, India: NCDIR-NCRP (ICMR); 2014 [Google Scholar].

15. Hussain MA, Pati S, Swain S, et al. Pattern and trends of cancer in Odisha, India: a retrospective study. Asian Pac J Cancer Prev APJCP. 2012;13(12):6333-6336.

16. Ugwu E, Obi S, Ezechukwu P, Okafor I, Ugwu A. Acceptability of human papilloma virus vaccine and cervical cancer screening among female health-care workers in Enugu, Southeast Nigeria. Niger J Clin Pract. 2013;16(2):249.

17. Ortashi O, Raheel H, Shalal M, Osman N. Awareness and knowledge about human papillomavirus infection and vaccination among women in UAE. Asian Pac J Cancer Prev APJCP. 2013;14(10):6077-6080.

18. Sabeena S, Bhat PV, Kamath V, Aswathyraj S, Arunkumar G. Knowledge, attitude and practice concerning human papilloma virus infection and its health effects among rural women, Karnataka, South India. Asian Pac J Cancer Prev APJCP. 2015;16(12):5053-5058.

19. Kulkarni SS, Kulkarni SS, Vastrad PP, et al. Prevalence and distribution of high risk human papillomavirus (HPV) Types 16 and 18 in Carcinoma of cervix, saliva of patients with oral squamous cell carcinoma and in the general population in Karnataka, India. Asian Pac J Cancer Prev APJCP. 2011:12(3):645-648.

20. Human Papilloma Virus ICMR. High Power Committee to Evaluate Performance of ICMR, 2012-2013. New Delhi, India: ICMR; 2014 Disease Specific Documents for XII plan.

21. Bansal AB, Pakhare AP, Kapoor N, Mehrotra R, Kokane AM. Knowledge, attitude, and practices related to cervical cancer among adult women: a hospital-based crosssectional study. J Nat Sci Biol Med. 2015 Dec;6(2):324-328.

22. Sharma J, Day P, Puroshottam R. Current understanding on HPV infection and cervical cancer along with risk factor among women of urban Delhi. Gynecol Oncol. 2013;118:S12-S17.

23. De Sanjose, Laimins L. A meta-analytic study on pathogenesis of human papillomaviruses. Microbiol Mol Biol. 2004;68:362-372.

24. Liu S, Chan K, Leung R, et al. Meta-analysis on the prevalence and risk factors of Human Papillomavirus (HPV) infection. PLoS One. 2013;6:e19244.

25. Zhao FH, Lewkowitz AK, Hu SY, et al. Prevalence of human papillomavirus and cervical intraepithelial neoplasia in China: a pooled analysis of 17 population-based studies. Int J Cancer. 2012;131:2929-2938.

26. Shi J, Canfell K, Lew J, et al. The burden of cervical cancer in China: synthesis of the evidence. Int J Cancer. 2012;130:641-652.

27. Li H, Thomas D, Jin S, et al. Tubal sterilization and use of an IUD and risk of cervical cancer. J Women's Health Gend Based Med. 2000;9:303-310.

28. Ma X, Wang J, Cheng Y, et al. Case-control study: the relationship between dietary factors and cervical cancer. Chin J Public Health. 2005;21:312-314.

29. Peng H, Liu S, Mann V, et al. Human papillomavirus types 16 and 33, herpes simplex virus type 2 and other risk factors for cervical cancer in Sichuan Province, China. Int J Cancer. 1991;47:711-716.

30. Wang Z, Chen Z, Wen H. Multivariate analysis of cervical cancer risk factors (100 case - control study). China Cancer. 1992;10:340-342. 